\title{
Polymerisation and Condensation Reactions
}

$\mathrm{A}^{\mathrm{T}}$

the meeting of the Faraday Society held on September 26-28 at Cambridge under the presidency of Mr. W. Rintoul, the subject of polymerisation and condensation reactions was discussed.

The technical development of the manufacture of the group of substances known as plastics, resins and moulding powders is now attaining large dimensions, and it occurred to the Colloid Committee of the Faraday Society that academic interest in these substances might be stimulated by such a meeting. In this it was not mistaken. Whilst the Faraday discussions have always been somewhat international in character, this meeting was favoured by the attendance of an unusual number of both members and visitors who came from overseas.

We are indebted in the first instance to Staudinger for emphasising the now generally accepted view that the polymers and condensates must be regarded as constituted of macromolecules formed by the polymerisation or condensation of single units. Both the physical and chemical properties of the finished materials should be capable of interpretation on this basis.

We now recognise three different types of macromolecules : the linear, which can be written $A(A)_{n} A$ or $(A B)_{n}$; the large ring or closed type; and the cross-linked variety. This latter in its simplest form might be regarded as a system of linear macromolecules linked together by crosslinkages like the rungs on a ladder, and in its most complex state as a crystal like silica.

Amongst a number of important and interesting topics three attracted most attention: the mechanism of growth of the large molecules from the small ones; the information yielded by $\mathrm{X}$-ray examination of the substances, especially when stressed; and the relationship between the mechanical and physical properties on one hand, and on the other the chemical structure of the material.

The discussion made it clear that there must be a number of different mechanisms by which macromolecules can be formed. For example, in the formation of a macromolecular ester from a dibasic acid and a dihydric alcohol, the chemical reaction involved, namely, the esterification process, would appear to be energetically identical both for commencing and for propagation of the ehain. On the other hand, in the polymerisation of a substance like styrene, the energetics of commencement and propagation are widely differ- ent. Once the growth has been initiated by the formation of what may be termed, to borrow from the nomenclature of chain reactions, a reaction centre, the chain propagation proceeds relatively rapidly.

During the course of the meeting, it became clear that a number of new factors not operative in the ordinary gaseous chain reaction must be considered in the growth of the macromolecule. For example, it is evident that in different systems the length of the macromolecule is subject to wide variation. In the case of many olefine polymerisation processes the mean length of the macromolecule corresponds to the dimer; in the case of the acrylic acid the length can certainly exceed ten thousand of the monomeric units. At present, it is difficult to elucidate the reason for this wide variation in chain length. It seems possible, at least in the olefines, that ring closure to form a cyclic compound, which can, of course, open again, plays an important part in retarding the growth of the chain. In the drying oils a steric factor appears to be important; the polymer must thus be regarded in its undistorted form as spherical rather than thread-like. Both in the polymerisation of acetylene and in the case of formaldehyde, it seems certain that chain termination is brought about by a special type of collision of the growing end of the chain with a monomer, the terminating collision differing from the propagating collision poth in the energy and the number of degrees of freedom involved.

One interesting feature of many of these reactions is the relatively rapid growth of the chain after initiation. It seems unlikely that this can take place through the ordinary collision mechanism, for the rate of diffusion of the monomer to the active centre at the end of the growing chain would then govern the reaction velocity; the energy of activation of the controlling process is known to be low, of the order of $5 \mathrm{kgm}$. cal. The suggestion was advanced that monomeric molecules might be supplied to the growing end of the chain by travelling along the chain. On this view, the process of chain growth must be regarded as analogous to the growth of crystals such as hydroquinone, where diffusion over the crystal surface plays a large part in controlling the rate of reaction.

Finally, it is well known that a macromolecule such as polystyrene can be decomposed into simple units by heat, and indeed at relatively low temperatures; the same appears to be true for 
the polymeric forms of formaldehyde, but many similar polymers are not so readily reversibly decomposed and require really high temperatures to bring about thermal destruction, from the products of which the monomer can be isolated. It is significant in this connexion that the pyrolysis of straight chain hydrocarbons proceeds more readily the longer the chain.

The chain-like structure of the cellobiose polymer cellulose and the more complicated polypeptide chain found in the proteins has been confirmed in a satisfactory manner by X-ray examination. These systems are, of course, quite distinct from the ordinary crystal in that the series of parallel chains along which the composition and structure are subject to repetition are usually so long that it is impossible to identify the relatively small elementary cell as determined by X-ray examination with a definite number of macromolecules. The molecules are much longer than the elementary cell and the X-ray pattern obtained is a fibre diagram.

A number of polymers like the polyoxymethylenes exhibit a crystalline pattern ; others like the phenol formaldehyde complexes only give an amorphous pattern; whilst a third and most interesting group give an amorphous pattern in their natural state but a crystalline pattern when stretched, these including rubber, polyvinyl alcohol, sulphur and polyethylene tetrasulphide. All these substances when stretched exhibit over a suitable range of temperature the remarkable extensibility shown by natural rubber. It is difficult to avoid the conclusion that great extensibility is associated with very long macromolecules, which are sufficiently flexible and at the same time mobile under stress to permit of orientation in parallel threads by the simple application of tension.

There are obvious difficulties to be overcome if this simple hypothesis be accepted as correct. On stretching rubber and freezing it, its fibre diagram and extension are both preserved; on warming, both extension and fibre diagram disappear, a process analogous to the melting of a solid. In a collection of small rod-shaped molecules in the liquid form, thermal agitation will ensure that the molecules are in complete disorder; but as the molecules grow longer the Van der Waals' forces of cohesion increase and the tendency for bundles or clusters of molecules to be formed with a common axis of orientation increases, a phenomenon termed cybotaxis. We might have anticipated unstretched rubber to be a cybotactic fluid par excellence. It is possible that this is really the case, but that the microclusters which are themselves in disorder are too small to be revealed by the X-rays. The thermal changes associated with the stretching of rubber give us a measure of the heat of adsorption of the polyprene chains on a polyprene surface.

The most important property of macromolecular polymers is that they alone amongst organic materials manifest to a significant degree such mechanical properties as strength, elasticity, toughness, pliability and hardness. Well oriented and fibrous polymers such as natural cellulose and silk are, weight for weight, stronger than iron. Synthetic polymers are not so strong as these natural products, and a good deal of attention has naturally been devoted to the problem of calculating the maximum tensile strength possible in a polymeric material and the cause of the relatively low values obtained. The following figures for the tensile strength in $\mathrm{kgm} . \mathrm{mm} .^{-2}$ for various materials at $20^{\circ} \mathrm{C}$. are interesting :

$\begin{array}{lrlr}\text { Best spring steel } & 197 & \text { Iron } & 20 \\ \text { Flax } & 100 & \text { Glass } & 5 \\ \text { Rubber } & 80 & \text { Polystyrene } & 4 \\ \text { Silk } & 35 & \text { Urea formaldehyde } & 3 \\ \text { Wool } & 28 & \text { Asphalt } & 1 \times 10^{-2} \\ \text { Artificial silk } & 25 & & \end{array}$

Calculations from the form of the Morse potential curve show that a tensile strength of no less than $2,000 \mathrm{kgm} . / \mathrm{mm}^{2}$ would be obtained for a completely orientated and fibrous rod composed of a bundle of macromolecules the individual lengths of which are equal to that of the rod. There seems little reason to doubt that materials stronger than the best steel should be capable of being made.

Another interesting property peculiar to these polymers is their extensibility or swelling in various solvents. Linear or chain-like polymers may be prepared which undergo simple solution in appropriate solvents.

As the chain length of the macromolecules increases, the process of molecular solution commences due to solvent imbition and swelling of the solid; this is followed by the formation of aggregates or micelles. These micelles are eventually dispersed to the molecular form on further appropriate dilution of the solution. On the other hand, cross-linked polymers such as silica or vulcanised rubber or phenol formaldehyde resins do not swell or disperse in solvents.

Staudinger has prepared a most interesting series of products from polystyrene, exhibiting all the transition stages. Polystyrene consists of simple thread-like macromolecules which will go into true solution in organic solvents ; the process of solution as we have noted being preceded by solvent imbition and swelling. If, however, to the styrene a small quantity of $p$-divinylbenzene be added before polymerisation, then when the chains are formed molecules of this material are incorporated in the growing chain and cross- 
linkages come into existence by the formation of divinylbenzene bridges. Such a cross-linked system is no longer dispersible in solvents, although if not too many cross-linkages are formed the material is capable of swelling. If the ratio of styrene to divinylbenzene is as high as 50,000 to 1 , the product is highly extensible but insoluble, whilst if it be as low as 1,000 to 1 the product shows scarcely any sign of swelling at all.

It is clear that the properties of the polymers and condensation products may be altered to a most extraordinary extent by the addition of suitable materials even in subanalytical amounts.

ERIC K. Rideal.

\section{Maintenance of Life in Isolated Animal Organs}

$\mathrm{T}^{\mathrm{H}}$ HE preliminary announcement of the work of Carrel and Lindberg on the maintenance of life in isolated organs, to which reference has already been made in our columns ${ }^{1}$, can now be amplified with further details of their method, from which an idea can be obtained of how the difficulties involved have been surmounted $^{2}$. The organs are removed with complete aseptic and antiseptic precautions from an animal that has just been killed by bleeding under anæsthesia; adult cats or fowls are generally used. With the organ are removed the surrounding tissues, arteries, veins, nerves and lymph vessels : for example, in the case of the ovary, with the organ itself are removed the Fallopian tube, and a flap of peritoneum and connective tissue containing the ovarian artery. During the operation the abdominal cavity and the organ are protected with gauze pads soaked in Dakin's solution.

The organ is introduced into the culture chamber protected by a sheet of 'cellophane' and the artery is connected with the cannula of the apparatus ; the chamber is closed by a rubber stopper and sealed with a cellulose acetate cement. A sterile pulsating circulation is maintained through the organ by the apparatus; different culture media have been used for this purpose. In order that the supply of glucose and bicarbonate may be sufficient for several days, the volume of the medium must be about 2,000 times greater than that of the tissues. For example, a cat's thyroid which weighs $85-110 \mathrm{mgm}$. requires about 230 c.c. of fluid. The apparatus may be used with 200 900 c.c. of medium. The latter may be bloodserum, diluted with Tyrode's solution, and containing sometimes hæmoglobin, or an artificial growth-activating solution, containing proteinsplit products, hæmin, cysteine, insulin, thyroxine, glutathione, vitamins $\mathrm{A}$ and $\mathrm{C}$, blood-serum, ete. A small amount of phenol red must be added, as indicator of the metabolic activity of the organ, as well as of the occurrence of bacterial infection. The medium is under an atmosphere of 40 per cent oxygen and 3-4 per cent carbon dioxide, the remainder being nitrogen, and is kept well aerated.
The apparatus is kept in an incubator at $37^{\circ}$ $38^{\circ} \mathrm{C}$. and is so designed that the organ and perfusion fluid can be continuously observed. The circulation is started about an hour after the death of the animal ; the number of pulsations is usually about 60 per minute, the systolic pressure being $120 \mathrm{~mm}$. mercury and the diastolic $60 \mathrm{~mm}$. mercury. Rate and pressure can be varied at will.

The organs used in the apparatus so far have been the thyroid gland, the ovary, the suprarenal gland, the spleen, heart and kidney. In the case of the thyroid, three different media have been used as perfusion fluids ; with diluted serum or an artificial medium containing amino-acids, the volume of the gland decreased and the follicles remained full of colloid, even after twenty-one days. With the medium containing peptones the glands grew and doubled their weight in three to four days; the consumption of sugar was increased more than three times. Histologically, there were observed either a disordered epithelial proliferation with disappearance of the colloid or an increase in the volume of the cells and in the number of follicles, with production of colloid. In an experiment with an ovary perfused with a growth-promoting medium, the weight of the organ increased from $90 \mathrm{mgm}$. to $284 \mathrm{mgm}$. in five days and three corpora lutea developed. Growth was, however, accompanied by disorganisation; there was a luxuriant and disordered proliferation of the stroma and of the epithelial cells.

These experiments show that an entire organ can be kept alive in vitro, increasing in size and weight, due to the appearance of new cells and tissues. The method should have great value in the study of different organs, especially those having an 'internal secretion', and further reports by Carrel and Lindberg will be awaited with interest. If it is permissible to speculate about the future, will it be possible for the chemist to utilise isolated perfused organs for the manufacture of their specific secretions in unlimited quantities, in cases in which a chemical synthesis is difficult or has not yet been achieved?

${ }^{1}$ NATURE, 135, 1066 ; 1935.

Science, 621, June 21, 1935. C.R. Acad. Sci., 14, July 1, 1935. 